\title{
Nonconvergence Results for the Application of Least-Squares Estimation to Ill-Posed Problems ${ }^{1}$
}

\author{
T. I. SEIDMAN ${ }^{2}$
}

Communicated by D. G. Luenberger

\begin{abstract}
One standard approach to solving $f(x)=b$ is the minimization of $\|f(x)-b\|^{2}$ over $x$ in $\tilde{\mathfrak{X}}$, where $\tilde{\mathfrak{X}}$ corresponds to a parametric representation providing sufficiently good approximation to the true solution $x^{*}$. Call the minimizer $x=\mathscr{A}(\tilde{\mathfrak{X}})$. Take $\tilde{\mathfrak{X}}=\mathfrak{X}_{N}$ for a sequence $\left\{\mathfrak{X}_{N}\right\}$ of subspaces becoming dense, and so determine an approximating sequence $\left\{x_{N}:=\mathscr{A}\left(\mathfrak{X}_{N}\right)\right\}$. It is shown, with $f$ linear and one-to-one, that one need not have $x_{N} \rightarrow x^{*}$ if $f^{-1}$ is not continuous.
\end{abstract}

Key Words. Ill-posed problems, least-squares estimation, approximation, parametric representation, convergence, nonconvergence.

\section{Introduction}

Many problems of practical importance involve the solution of equations

$$
f(x)=b
$$

in which the unknown is an element of some infinite-dimensional space $\mathfrak{X}$ (e.g., a function or set of functions). For example, one might seek to determine a coefficient function in a partial differential equation from observational data taken from a solution; here, $b$ denotes the observation and $x$ would denote the unknown coefficient function, together with such other data as must be adjoined to what is known already to make a

\footnotetext{
${ }^{1}$ This work was supported by the US Army Research Office under Grant No. DAAG-29-77G-0061. The author is indebted to the late W. C. Chewning for suggesting the topic in connection with computing optimal boundary controls for the heat equation (Ref. 2).

${ }^{2}$ Associate Professor, Department of Mathematics, University of Maryland Baltimore County, Baltimore, Maryland.
} 
well-defined map: $x \mapsto b$. For more, related applications, see e.g. Refs. $1,3,6$.

If $b$ is to be given (in practice, only approximately) as an element of a certain space $\mathfrak{V}$, then it may happen that $f$ has no continuous inverse. In that case, we say that the problem (1) is ill posed. We assume that only the continuity of $f^{-1}$ is at issue, i.e., that $f$ is one-to-one and that the given $b$ is in the range of $f$.

It is clear that one may attempt to solve (1) by minimizing the square of the residual .error, replacing (1) by the optimization problem:

$$
\text { minimize } \mathscr{F}(x):=\frac{1}{2}\|f(x)-b\|_{\mathfrak{Y}}^{2} \quad \text { over } x \text { in } \mathfrak{X} .
$$

Since any feasible computation must be finitary, a standard approach is to assume a parametric representation for $x$, treat $\mathscr{J}$ as a function of the finite number of parameters, and use (2) to estimate the parameter values and so determine $x$. Typical representations might be spline approximations or truncated power series or Fourier series expansions.

For simplicity of analysis, we shall assume that the problem is linear,

$$
f(x):=A x,
$$

and that the spaces $\mathfrak{X}, \mathfrak{Y}$ are Hilbert spaces. The approach described above now consists of determining $x$ by solving the finite-dimensional quadratic optimization problem:

$$
\text { minimize } \mathscr{J}(x):=\frac{1}{2}\|A x-\tilde{b}\|_{\mathfrak{Y}}^{2} \quad \text { over } x \text { in } \tilde{\mathfrak{X}},
$$

where $\tilde{\mathfrak{X}}$ is a finite-dimensional subspace of $\mathfrak{X}$ and $\tilde{b}$ is the actual observation,

$$
\tilde{b} \approx b^{*}:=A x^{*} \text {. }
$$

Here, $x^{*}$ denotes the true solution, and $b^{*}$ the corresponding true observational data. Note that, even if $b^{*}$ were somehow available exactly, computational imprecision and the exigencies of finitary representation would introduce such potential perturbation. The approximant determined by (3) is denoted by $\mathscr{A}(\tilde{X})$.

As with most computational schemes, one applies (3) but analyzes the procedure asymptotically, embedding $\tilde{\mathfrak{X}}$ as one of an increasing family of subspaces $\left\{\mathfrak{X}_{N}\right\}$ and $\tilde{b}$ as one of an approximating sequence $\left\{b_{N}\right\}$. Such a scheme would be termed convergent if $b_{N} \rightarrow b^{*}$ implies that $x_{N} \rightarrow x^{*}$, where each $x_{N}=\mathscr{A}\left(\mathfrak{X}_{N}\right)$ is the solution of the following problem:

$$
\operatorname{minimize} \mathscr{J}_{N}(x):=\frac{1}{2}\left\|A x-b_{N}\right\|_{\mathscr{V}}^{2} \quad \text { over } x \text { in } \mathfrak{X}_{N} \text {. }
$$

If $A$ is one-to-one, then (4) always has a unique solution, and it is easy to see that this solution is just $A^{-1}$ acting on the orthogonal projection of $b_{N}$ on the 
finite-dimensional subspace

$$
\mathfrak{Y}_{N}:=A \mathfrak{X}_{N} \subset \mathfrak{Y} \text {. }
$$

If $A$ has a continuous inverse, it is clear that the scheme (4) is convergent, since the sequence of projections onto $\left\{\mathfrak{V}_{N}\right\}$ converges strongly to the identity. On the other hand, our aim in this paper is to show that this is always false if the problem is ill posed, i.e., if $A^{-1}$ is unbounded. There are two principal results in that case.

(i) For any $\left\{\mathfrak{X}_{N}\right\}$ and any $b^{*}=A x^{*}$, there exist sequences $b_{N} \rightarrow b^{*}$ such that $\left\|x_{N}\right\| \rightarrow \infty$ and also such that $\left\{x_{N}=\mathscr{A}\left(\mathfrak{X}_{N}\right)\right\}$ is bounded, but $x_{N} \nrightarrow x^{*}$. If $\left\{x_{N}\right\}$ is bounded, one always has weak convergence: $x_{N} \rightarrow x^{*}$.

(ii) Even if $b^{*}$ would be given exactly $\left(b_{N}=b^{*}=A x^{*}\right.$ for each $\left.N\right)$, for almost any such $b^{*}$ there exist $\left\{\mathfrak{X}_{N}\right\}$ for which $\left\{x_{N}=\mathscr{A}\left(\mathfrak{X}_{N}\right)\right\}$ is unbounded.

The first of these results is unsurprising; after all, the instability of the solution under perturbation of the data is characteristic of ill-posed problems. Only its simplicity in use and its usefulness in the well-posed case can account for the otherwise inexplicable persistence of this approach in engineering practice, despite its obvious shortcomings (even after reduction to the parametrized formulation; see the discussion and references in Ref. 1). It is the second result which is somewhat astonishing; even with exact data (and arbitrarily rapid convergence of the eigenfunction expansion of the right-hand side), one cannot be confident of convergence or even of boundedness of the sequence of computed approximants.

These results indicate the necessity for extreme caution in dealing with ill-posed problems. For a discussion of some convergent computational approaches to ill-posed problems, see Ref. 6. Note that, for particular classes of applications, such projection-estimation schemes, with $\left\{\mathfrak{X}_{N}\right\}$ of specified form, can be justified (see Refs. 4, 5). It is the necessity of doing this which is implied by the present results.

\section{Nonconvergence with Perturbation}

As above, let $\mathfrak{X}$ and $\mathfrak{Y}$ be infinite-dimensional Hilbert spaces; and let $A_{0}: \mathfrak{X} \rightarrow \mathfrak{Y}$ be compact, injective, and with dense range (this last hypothesis is not significant; otherwise, replace $\mathfrak{Y}$ by the closure of the range). Let the eigenvalues of $\boldsymbol{A}_{0}^{*} \boldsymbol{A}_{0}: \mathfrak{X} \rightarrow \mathfrak{X}$, taken with multiplicities, be $\left\{\alpha_{1}^{2}, \alpha_{2}^{2}, \ldots\right\}$; and let $\left\{a_{1}, a_{2}, \ldots\right\}$ be the corresponding eigenvectors. The eigenvalues are positive (we also take $\alpha>0$ ) and converge to zero; assume that they are ordered decreasingly so $\alpha_{1} \geq \alpha_{2} \ldots$. The eigenvectors may be taken to form an orthonormal basis of $\mathfrak{X}$. 
Now, define $U: \mathfrak{X} \rightarrow \mathfrak{Y}$ such that

$$
U a_{n}=\alpha_{n}^{-1} A_{0} a_{n} .
$$

It is easily verified that $\left\{U a_{n}\right\}$ is orthonormal, so $U$ is unitary and, as $A_{0}$ has dense range, is surjective. Let

$$
A:=U^{*} A_{0}: \mathfrak{X} \rightarrow \mathfrak{X},
$$

and observe that $A$ is self-adjoint, with eigenvalues $\left\{\alpha_{n}\right\}$ and eigenvectors $\left\{a_{n}\right\}$, compact, injective, and has dense range. Note that, for any $x \in \mathfrak{X}$, $y_{0} \in \mathfrak{V}$, one has

$$
\left\|A_{0} x-y_{0}\right\|_{\mathfrak{Y}}=\|A x-y\|_{x}
$$

where

$$
y:=U^{*} y_{0}
$$

We have thus shown that, with no loss of generality, it is always possible to reduce considerations to positive self-adjoint operators, such as $A: \mathfrak{X} \rightarrow \mathfrak{X}$. Henceforth, we assume that $A_{0}$ is already in the form of $A$, and we omit the subscript on the norm as only one space is involved.

Consider an arbitrary increasing sequence of subspaces $\left\{\mathfrak{X}_{N}\right\}$, with $\cup_{N} \mathfrak{X}_{N}$ dense in $\mathfrak{X}$. Assume, for simplicity, that

$$
\operatorname{dim} \mathfrak{X}_{N}=N \text {, }
$$

so there is an orthonormal basis $\left\{e_{1}, e_{2}, \ldots\right\}$ of $\mathfrak{X}$ such that

$$
\mathfrak{X}_{N}=\operatorname{sp}\left\{e_{1}, \ldots, e_{N}\right\} \text {. }
$$

Let

$$
\mathfrak{Y}_{N}:=A \mathfrak{X}_{N} \subset \mathfrak{X},
$$

and let

$$
A_{N}: \mathfrak{X}_{N} \rightarrow \mathfrak{V}_{N}
$$

be the restriction of $A$. Clearly, $A_{N}$ is invertible, and we set

$$
\nu_{N}:=\left\|A_{N}^{-1}\right\| .
$$

It can be shown (see e.g. Lemma 4.6 of Ref. 2) that

$$
\nu_{N} \geq 1 / \alpha_{N},
$$

so $\nu_{N} \rightarrow \infty$. 
Theorem 2.1. Let $A,\left\{\mathfrak{X}_{N}\right\}$, etc., be as above. Let $x^{*} \in \mathfrak{X}$ and $b^{*}:=A x^{*}$. Then, there exists a sequence $b_{N} \rightarrow b^{*}$ for which the corresponding sequence $\left\{x_{N}=\mathscr{A}\left(\mathfrak{X}_{N}\right)\right\}$, given by (4), has $\left\|x_{N}-x^{*}\right\| \rightarrow \infty$.

Note that, although we have written just $\mathscr{A}\left(\mathfrak{X}_{N}\right), x_{N}$ depends on $b_{N}$ as well as on the choice of $\mathfrak{X}_{N}$.

Proof. There exists $v_{N}$ in $\mathfrak{Y}_{N}$, with

$$
\left\|v_{N}\right\|=\nu_{N}^{-1 / 2}
$$

such that

$$
v_{N}=A u_{N}
$$

i.e.,

$$
u_{N}:=A_{N}^{-1} v_{N} \in \mathfrak{X}_{N}
$$

with

$$
\left\|u_{N}\right\|=\nu_{N}^{1 / 2} .
$$

Let $\tilde{b}_{N}$ be the orthogonal projection of $b^{*}$ on $\bigvee_{N}$ and

$$
\tilde{x}_{N}:=A_{N}^{-1} \tilde{b_{N}}
$$

$\tilde{x}_{N}$ is what one would obtain from (4) using $b_{N}=b^{*}$. One of $\left\|\tilde{x}_{N} \pm u_{N}\right\|$ is greater than $\left\|u_{N}\right\|$; and, with that choice of sign, take

$$
b_{N}:=b^{*} \pm v_{N} \text {. }
$$

Then,

$$
x_{N}=A_{N}^{-1}\left(\tilde{b}_{N} \pm v_{N}\right)=\tilde{x}_{N} \pm u_{N}
$$

so

$$
\left\|x_{N}\right\| \geq\left\|u_{N}\right\| \rightarrow \infty
$$

whence

$$
\left\|x_{N}-x^{*}\right\| \rightarrow \infty
$$

while

$$
\left\|b_{N}-b^{*}\right\| \leq\left\|b_{N}-\tilde{b_{N}}\right\|+\left\|\tilde{b_{N}}-b^{*}\right\| \rightarrow 0
$$

since

$$
\left\|\tilde{b_{N}}-b_{N}\right\|=\left\|v_{N}\right\| \rightarrow 0,
$$

and the projections on $\mathfrak{V}_{N}$ go strongly to the identity as $A$ has dense range and $\bigcup_{N} \mathfrak{X}_{N}$ is dense. 
To construct $b_{N} \rightarrow b^{*}$, with $\left\{x_{N}\right\}$ bounded but not converging to $x^{*}$, let $\hat{x}_{N}$ be the orthogonal projection of $x^{*}$ on $\mathfrak{X}_{N}$, and then set

$$
b_{N}:=A \hat{x}_{N}+\hat{v}_{N}
$$

where

$$
\hat{v}_{N}=A \hat{u}_{N} \in \mathfrak{V}_{N}
$$

has

$$
\left\|\hat{u}_{N}\right\|=1, \quad\left\|\hat{v}_{N}\right\|=1 / \nu_{N}
$$

i.e.,

$$
\hat{v}_{N}=\nu_{N}^{-1 / 2} v_{N}
$$

Since $\hat{x}_{N} \in \mathfrak{X}_{N}$, we have

$$
x_{N}=\hat{x}_{N}+\hat{u}_{N} .
$$

Since $\hat{x}_{N} \rightarrow x^{*}$, we have

$$
\left\|x_{N}-x^{*}\right\| \rightarrow 1=\left\|u_{N}\right\|
$$

so $x_{n} \nrightarrow x^{*}$. Also,

$$
A \hat{x}_{N} \rightarrow A x^{*}=b^{*} \quad \text { and } \quad \hat{v}_{N} \rightarrow 0 \text {, }
$$

so $b_{N} \rightarrow b^{*}$.

For any $b_{N}$ one has

$$
A x_{N}=\tilde{b_{N}},
$$

the projection of $b_{N}$ on $\mathfrak{V}_{N}$, so $b_{N} \rightarrow b^{*}$ implies

$$
\tilde{b_{N}}=A x_{N} \rightarrow b^{*} \text {. }
$$

If any subsequence of $\left\{x_{N}\right\}$ were bounded, then any further subsequence would contain a weakly convergent subsequence, $x_{N(k)} \rightarrow x_{*}$. Since $A x_{N(k)} \rightarrow$ $b^{*}$ and the graph of $A$ is closed in the weak topology of $\mathfrak{X} \times \mathfrak{X}$, one has

$$
A x_{*}=b^{*} \text {, }
$$

so $x_{*}=x^{*}$. Thus, the original subsequence converges to $x^{*}$ weakly, although as shown, not necessarily strongly.

\section{Nonconvergence with Exact Data}

We first show by an example that, even using the unperturbed data $b^{*}$, one may encounter the behavior noted above. Let $\left\{\mathfrak{X}_{N}=\operatorname{sp}\left\{e_{1}, \ldots, e_{N}\right\}\right\}$ be 
any increasing sequence of subspaces as above, $\left\{e_{n}\right\}$ an orthonormal basis of the Hilbert space $\mathfrak{X}$. We wish to construct

$$
A_{0}: \mathfrak{X} \rightarrow \mathfrak{X} \text { and } b^{*}=A_{0} x^{*}
$$

(recalling the beginning of Section 2, we write $A_{0}$, since it is convenient here not to assume a reduction to the self-adjoint case) for which $\left\{x_{N}=\mathscr{A}\left(\mathfrak{X}_{N}\right)\right\}$, as computed by (4) using the exact right-hand side $b_{N}=b^{*}$ for each $N$, has $\left\|x_{N}-x^{*}\right\| \rightarrow \infty$.

Example 3.1. We take $A_{0}$ to be given in the form

$$
A_{0}: \sum_{n=1}^{\infty} \xi_{n} e_{n} \mapsto \sum_{n=1}^{\infty}\left(\alpha_{n} \xi_{n}+\beta_{n} \xi_{1}\right) e_{n}
$$

with

$$
\alpha_{1}=1, \quad \beta_{1}=0, \quad \sum \beta_{n}^{2}<\infty, \quad 0 \neq \alpha_{n} \rightarrow 0 .
$$

This defines a compact, injective linear operator $A_{0}: \mathfrak{X} \rightarrow \mathfrak{X}$ with dense range. Writing

we have

$$
x^{*}=\sum_{1}^{\infty} \xi_{n}^{*} e_{n}, \quad x=\sum_{1}^{\infty} \xi_{n} e_{n}
$$

$$
\mathscr{J}(x)=\frac{1}{2}\left\|A_{0}\left(x-x^{*}\right)\right\|^{2}=\frac{1}{2} \sum_{n=1}^{\infty}\left[\alpha_{n}\left(\xi_{n}-\xi_{n}^{*}\right)+\beta_{n}\left(\xi_{1}-\xi_{1}^{*}\right)\right]^{2}
$$

in (4).

Taking $x=\mathscr{A}\left(\mathfrak{X}_{N}\right)$, one has

$$
0=\partial \mathscr{J} / \partial \xi_{n}=\alpha_{n}\left[\alpha_{n}\left(\xi_{n}-\xi_{n}^{*}\right)+\beta_{n}\left(\xi_{1}-\xi_{1}^{*}\right)\right], \quad \text { for } n=2, \ldots, N ;
$$

and, using (6), and noting that $\xi_{m}=0$ for $n>N$,

$$
0=\partial \mathscr{F} / \partial \xi_{1}=\left(\xi_{1}-\xi_{1}^{*}\right)+\sum_{n=N+1}^{\infty} \beta_{n}\left[\alpha_{n}\left(-\xi_{n}^{*}\right)+\beta_{n}\left(\xi_{1}-\xi_{1}^{*}\right)\right],
$$

so

$$
\xi_{1}-\xi_{1}^{*}=\left[\sum_{N+1}^{\infty} \alpha_{n} \beta_{n} \xi_{n}^{*}\right] /\left[1+\sum_{N+1}^{\infty} \beta_{n}^{2}\right] .
$$

Thus, using (6) and (7),

$$
\begin{aligned}
\left\|x_{N}-x^{*}\right\|^{2} & =\sum_{1}^{N}\left(\xi_{n}-\xi_{n}^{*}\right)^{2}+\sum_{N+1}^{\infty} \xi_{n}^{* 2} \\
& =\left[1+\sum_{2}^{N}\left(\beta_{n} / \alpha_{n}\right)^{2}\right]\left[\sum_{N+1}^{\infty} \alpha_{n} \beta_{n} \xi_{n}^{*}\right] /\left[1+\sum_{N+1}^{\infty} \beta_{n}^{2}\right]+\sum_{N+1}^{\infty} \xi_{n}^{* 2} .
\end{aligned}
$$


Suppose, now, that $\left\{\beta_{n}\right\}_{2}^{\infty}$ and $\left\{\xi_{n}^{*}\right\}_{1}^{\infty}$ have been given arbitrarily, but with

$$
\sum_{n} \beta_{n}^{2}<\infty, \quad \sum_{n} \xi_{n}^{* 2}<\infty,
$$

and all terms nonzero. We wish to construct $\left\{\alpha_{n}\right\}_{2}^{\infty}$, so

$$
0 \neq \alpha_{n}, \quad \alpha_{n} \rightarrow 0, \quad\left\|x_{N}-x^{*}\right\| \rightarrow \infty .
$$

For example, take $\alpha_{n}=1 / n$ for $n$ even; and, for $n$ odd, take $\alpha_{n} \leq 1 / n$ and so small that

$$
\left(\beta_{n} / \alpha_{n}\right)\left(\alpha_{n+3} \beta_{n+3} \xi_{n+3}^{*}\right) \geq(n+1)^{2}\left[1+\sum_{2}^{\infty} \beta_{n}^{2}\right] .
$$

Clearly, the use of (9) in (8) for $N=n, n+1$ gives

$$
\left\|x_{N}-x^{*}\right\| \geq N
$$

so

$$
\left\|x_{N}-x^{*}\right\| \rightarrow \infty
$$

as desired for this operator $A_{0}$. It is somewhat more delicate to arrange that

$$
\left\|x_{N}-x^{*}\right\| \rightarrow c \neq 0, \infty
$$

but this can also be done.

We now return to the notation of the self-adjoint case. Thus, $A$ is now assumed to have an orthonormal basis $\left\{a_{n}\right\}_{1}^{\infty}$ of eigenvectors with positive eigenvalues $\left\{\alpha_{n}\right\}$.

Theorem 3.1. Let $A$ be a compact, injective linear operator with dense range. Let $b^{*}:=A x^{*}$ be given, with $x^{*}$ not a finite linear combination of eigenvectors. Then, there is an orthonormal basis $\left\{e_{n}\right\}$ of $\mathfrak{X}$ such that the sequence $x_{N}=\mathscr{A}\left(\mathfrak{X}_{N}\right)$, determined using (4) with

$$
\mathfrak{X}_{N}:=\operatorname{sp}\left\{e_{1}, \ldots, e_{N}\right\} \quad \text { and } b_{N}=b^{*}
$$

for each $N$, is unbounded.

Proof. The construction presented here gives

$$
\left\|x_{2 J-1}\right\| \rightarrow \infty,
$$

but $x_{2 J} \rightarrow x^{*}$, under the simplifying assumption that

$$
x^{*}=\sum_{1}^{\infty} \beta_{n}^{*} a_{n},
$$


with $\beta_{n} \neq 0$ for every $n$. It is not too difficult to modify the construction to admit having infinitely many $\beta_{n}=0$, provided also that infinitely many $\beta_{n} \neq 0$.

The eigenvalues and eigenvectors are given with some ordering, and we start by reordering them recursively to suit our purposes. At each step, let $S_{m}$ be the set of indices as yet not chosen when one comes to select the $m$ th in the new ordering; we proceed to choose in the order: $m=2,1,4$, $3,6,5, \ldots, 2 j, 2 j-1, \ldots$ For $m=2 j$, let $n(2 j)$ be simply the least $n$ in $S_{2 j}$. For $m=2 j-1$, let $n(2 j-1)$ be the least $n$ in $S_{2 j-1}:=S_{2 j} \mid\{n(2 j)\}$ for which $\alpha_{n}$ is small enough that

$$
\rho_{j}:=\left[\alpha_{n} / \alpha_{n(2 j)}\right]<\min \left\{\left|\beta_{n(2 j)}^{*}\right| / j, 1 / \sqrt{ } 2\right\} .
$$

For simplicity of notation, we now simply write $\hat{a}_{m}, \hat{\alpha}_{m}, \hat{\beta}_{m}^{*}$ for $a_{n(m)}$, $\alpha_{n(m)}, \beta_{n(m)}^{*}$, assuming the new ordering. Thus,

$$
0<\rho_{i}:=\hat{\alpha}_{2 j-1} / \hat{\alpha}_{2 j}<\left|\hat{\beta}_{2 j}^{*}\right| / j, \quad 1-\rho_{i}^{2} \geq 1 / 2,
$$

for $j=1,2, \ldots$ Now, set

$$
s_{j}= \pm \rho_{j}, \quad c_{j}=\sqrt{ }\left(1-\rho_{j}^{2}\right) \quad \text { with } \quad s_{i} \hat{\beta}_{2 j-1}^{*} \hat{\beta}_{2 j}^{*}>0,
$$

and then define a new basis $\left\{e_{n}\right\}$ in terms of $\left\{\hat{a}_{m}\right\}$ by

$$
e_{2 j-1}:=c_{j} \hat{a}_{2 j-1}-s_{j} \hat{a}_{2 j}, \quad e_{2 j}:=s_{j} \hat{a}_{2 j-1}+c_{j} \hat{a}_{j} \text {. }
$$

Note that, since

$$
c_{i}^{2}+s_{j}^{2}=1 \quad \text { for each } j,
$$

$\left\{e_{n}\right\}_{1}^{\infty}$ is again an orthonormal basis. We will take

$$
\mathfrak{X}_{N}:=\operatorname{sp}\left\{e_{1}, \ldots, e_{N}\right\} \quad \text { and } \quad x_{N}=\mathscr{A}\left(\mathfrak{X}_{N}\right) .
$$

Expansions with respect to the bases $\left\{\hat{a}_{m}\right\},\left\{e_{n}\right\}$ are given by

$$
x=\sum_{m} \beta_{m} \hat{a}_{m}=\sum_{n} \gamma_{n} e_{n}
$$

with

$$
\begin{array}{ll}
\gamma_{2 j-1}=c_{j} \beta_{2 j-1}-s_{j} \beta_{2 j}, & \gamma_{2 j}=s_{i} \beta_{2 j-1}+c_{j} \beta_{2 j}, \\
\beta_{2 j-1}=c_{j} \gamma_{2 j-1}+s_{j} \gamma_{2 j}, & \beta_{2 j}=-s_{j} \gamma_{2 j-1}+c_{j} \gamma_{2 j} .
\end{array}
$$

Since we take

$$
b^{*}=A x^{*},
$$

so

$$
A x-b^{*}=A\left(x-x^{*}\right)
$$


we have for consideration in (4):

$$
\begin{aligned}
\mathscr{J}(x):= & \frac{1}{2}\left\|A\left(x-x^{*}\right)\right\|^{2}=\frac{1}{2}\left\|\sum_{k}\left(\beta_{k}-\hat{\beta}_{k}^{*}\right) \hat{\alpha}_{k} \hat{a}_{k}\right\|^{2} \\
= & \frac{1}{2} \sum_{j=1}^{\infty}\left\|\left(\gamma_{2 j-1}-\gamma_{2 j-1}^{*}\right) A e_{2 j-1}+\left(\gamma_{2 j}-\gamma_{2 j}^{*}\right) A e_{2 j}\right\|^{2} \\
= & \frac{1}{2} \sum_{1}^{\infty}\left[\left(c_{i}\left[\gamma_{2 j-1}-\gamma_{2 j-1}^{*}\right]+s_{j}\left[\gamma_{2 j}-\gamma_{2 j}^{*}\right]\right)^{2} \hat{\alpha}_{2 j-1}^{2}\right. \\
& \left.+\left(-s_{j}\left[\gamma_{2 j-1}-\gamma_{2 j-1}^{*}\right]+c_{j}\left[\gamma_{2 j}-\gamma_{2 j}^{*}\right]\right)^{2} \hat{\alpha}_{2 j}^{2}\right] .
\end{aligned}
$$

For minimizing $\mathscr{J}$, we consider $\partial \mathscr{J} / \partial \gamma_{n}$. With some manipulation,

$$
\begin{aligned}
\partial \mathscr{J} / \partial \gamma_{2 j-1} & =\left[c_{j}^{2} \hat{\alpha}_{2 j-1}^{2}+s_{j}^{2} \hat{\alpha}_{2 j}^{2}\right]\left(\gamma_{2 j-1}-\gamma_{2 j-1}^{*}\right)+\left[\hat{\alpha}_{2 j-1}^{2}-\hat{\alpha}_{2 j}^{2}\right] s_{j} c_{j}\left(\gamma_{2 j}-\gamma_{2 j}^{*}\right), \\
\partial \mathscr{J} / \partial \gamma_{2 j} & =\left[\alpha_{2 j-1}^{2}-\hat{\alpha}_{2 j}^{2}\right] s_{j} c_{j}\left(\gamma_{2 j-1}-\gamma_{2 j-1}^{*}\right)+\left[s_{j}^{2} \hat{\alpha}_{2 j-1}^{2}+c_{j}^{2} \hat{\alpha}_{2 j}^{2}\right]\left(\gamma_{2 j}-\gamma_{2 j}^{*}\right) .
\end{aligned}
$$

If both of these expressions vanish [e.g., for $x=x_{N}=\mathscr{A}\left(\mathfrak{X}_{N}\right)$ with $N \geq 2 j$ ], then

$$
\gamma_{2 j-1}=\gamma_{2 j-1}^{*} \quad \text { and } \quad \gamma_{2 j}=\gamma_{2 j}^{*}
$$

since the determinant of the system is

$$
\hat{\alpha}_{2 j-1}^{2} \hat{\alpha}_{2 j}^{2} \neq 0 \text {. }
$$

For $N=2 J$, this makes $x_{2 J}$ the projection of $x^{*}$ on $\mathfrak{X}_{2 J}$, so the subsequence $\left\{x_{2 J}\right\}$ will converge to $x^{*}$.

Suppose, now, that

$$
N=2 J-1,
$$

on the other hand. One has

$$
\gamma_{n}=\gamma_{n}^{*} \quad \text { for } n<2 J-1=N ;
$$

but, for

$$
n=2 J-1,
$$

one has

$$
\partial \mathscr{J} / \partial \gamma_{2 J-1}=0
$$

in (14), with $j=J$, but must have $\gamma_{2 J}=0$ as $\gamma_{n}=0$ for $n>N$. Thus,

$$
\begin{aligned}
\gamma_{2 J-1}-\gamma_{2 J-1}^{*} & =\frac{\left[\hat{\alpha}_{2 J-1}^{2}-\hat{\alpha}_{2 J}^{2}\right] s_{J} c_{J}}{c_{J}^{2} \hat{\alpha}_{2 J-1}^{2}+s_{J}^{2} \hat{\alpha}_{2 J}^{2}} \gamma_{2 J}^{*} \\
& =\frac{\left[\rho_{J}^{2}-1\right] s_{J_{J}}}{\left[\rho_{J}^{2}-1\right] c_{J}^{2}+1}\left[s_{J} \hat{\beta}_{2 J-1}^{*}+c_{J} \hat{\beta}_{2 J}^{*}\right],
\end{aligned}
$$


and so

$$
\begin{aligned}
\left(\gamma_{2 J-1}-\gamma_{2 J-1}^{*}\right)^{2} & =\frac{\left[\rho_{J}^{2}-1\right]^{2} \rho_{J}^{2}\left(1-\rho_{J}^{2}\right)}{\left(\left[\rho_{J}^{2}-1\right]\left(1-\rho_{J}^{2}\right)+1\right)^{2}}\left[ \pm \rho_{J} \hat{\beta}_{2 J-1}^{*}+\sqrt{ }\left(1-\rho_{J}^{2}\right) \hat{\beta}_{2 J}^{*}\right]^{2} \\
& >\hat{\beta}_{2 J}^{* 2} / 64 \rho_{J}^{2}>J^{2} / 64
\end{aligned}
$$

using (10-2), (11), (13). Thus,

$$
\left\|x_{2 J-1}-x^{*}\right\| \geq J / 8
$$

and the sequence $\left\{x_{N}\right\}$ is unbounded $\left(\left\|x_{2 j-1}\right\| \rightarrow \infty\right)$.

It is easy to see from (15) that replacing (11) by a different choice of $\left(s_{J}, c_{J}\right)$, still subject to

$$
s_{I}^{2}+c_{J}^{2}=1,
$$

permits construction of a new basis $\left\{e_{n}\right\}$ giving to $\left|\gamma_{2 J-1}-\gamma_{2,-1}^{*}\right|$ an arbitrary value in the interval $[0, J / 8]$ without affecting the results of any other computations, i.e., results for $x_{N}$ with $N$ other than $2 J-1$. Thus, one can also arrange that $\left\|x_{2 J-1}-x *\right\| \rightarrow c$ for any choice of $c$ in $[0, \infty]$; so, in particular, one can have $\left\{x_{N}\right\}$ bounded with $x_{N} \nrightarrow x^{*}$.

It is clear that the construction above requires $\beta_{n}^{*} \neq 0$ for infinitely many $n$ for (10-2) to be possible. If $x^{*} \neq 0$, then it seems likely that a suitable orthonormal basis $\left\{e_{n}\right\}$, not of the form (12) or indeed block-related to $\left\{\alpha_{k}\right\}$ at all, could be found, say of the form

$$
e_{n}=\sum_{1}^{n} c_{n, j} \hat{a}_{j}
$$

with $c_{n, n} \neq 0$ and $\left\{a_{n}\right\}$ a suitable reordering of $\left\{a_{k}\right\}$, for which the expansion coefficients $\left\{\left\langle x^{*}, e_{n}\right\rangle\right\}$ decay slowly enough and (4) gives $\left\{\left\|x_{N}-x^{*}\right\|\right\}$ unbounded or even, more strongly, $\left\|x_{N}-x^{*}\right\| \rightarrow \infty$. We have preferred here to present Theorem 3.1, rather than to cope with the computational complexities of this more general form.

\section{Discussion}

The use of computations based on an assumed (approximate) parametric representation for an unknown function to be estimated is pervasive in engineering practice and system theory. Indeed, the very term lumped parameter indicates such an approach to system structure. The use of minimization of the residual is then a standard approach to estimation of the parameter values. 
The results above show that, for ill-posed problems, this is an unacceptable procedure in the absence of detailed justificatory analysis of $\left\{A_{N}^{-1}\right\}$ and, for the particular $b^{*}$ involved, of the convergence to $b^{*}$ of the projections $\tilde{b}_{N}$ on $\mathfrak{V}_{N}:=A \mathfrak{X}_{N}$. The analysis presented was only for linear problems (whereas, e.g., system identification problems are nonlinear even in the case of linear dynamics). However assuming, as is typically done, that $f$ in (1) is smoothly Fréchet differentiable near the desired solution $x^{*}$, convergence to $x^{*}$ of the approximating sequence $\left\{x_{N}\right\}$ would suggest applicability of the linearized model, and so would suggest the relevance of the results above. At present, no rigorous realization of this argument is available, even for Theorem 2.1 under strong smoothness and uniformity hypotheses on the Fréchet derivative. It is equally true, of course, that the present results preclude the possibility of any justification via linearization of the convergence of the algorithm:

$$
\text { minimize } \mathscr{F}_{N}(\lambda):=\frac{1}{2}\left\|f\left(x_{n}(\lambda)\right)-b_{N}\right\|^{2} \quad \text { over } \lambda \in \Lambda_{N},
$$

where $\left\{\Lambda_{N}\right\}$ is a sequence of parameter spaces and $\left\{\lambda \mapsto x_{N}(\lambda)\right\}$ are the corresponding parametrizations; now,

$$
\mathfrak{X}_{N}:=\left\{x_{N}(\lambda): \lambda \in \Lambda_{N}\right\}
$$

need no longer be a linear subspace but is locally diffeomorphic to $\Lambda_{N}$.

It should be emphasized that it is not the method of least squares per se which is causing the problem. ${ }^{3}$ The real difficulty ${ }^{4}$ lies with the use of approximating subspaces which may be poorly related to the operator $A$. The constructions of Example 3.1 and Theorem 3.1 are, of course, quite artificial and one might expect (and would hope) that natural choices of subspaces would (as e.g. in Ref. 5) lead to convergent approximation sequences. On the other hand, the existence of even such artificial constructions makes that expectation and hope less confident and emphasizes the need for careful examination of the procedure.

In particular, for ill-posed problems, it is inadequate to verify that particular computational procedures apply to effective treatment of (1) or

\footnotetext{
${ }^{3}$ Another popular approximation method (for $A$ positive) selects $x_{N}$ in $\mathfrak{X}_{N}$ to make the residual $A x_{N}-b$ orthogonal to $\mathfrak{X}_{N}$ and an essentially identical construction can be used to demonstrate the possibility of nonconvergence for that method. On the other hand, if $\mathfrak{X}_{N}$ were in the range of $A^{*}$, one could select $x_{N}$ in $x_{N}$ to make $A x_{N}-b$ orthogonal to $\mathfrak{Y}_{N}$, where $\mathfrak{X}_{N}=A^{*} \mathfrak{Y}_{N}$ and the $x_{N}$ so selected will be the best approximation in $\mathfrak{X}_{N}$ to the true solution.

${ }^{4}$ The instability under perturbation exhibited in Theorem 2.1 is inherent in the ill-posedness of the problem, but is not the real difficulty. If the difficulty exhibited in Example 3.1 and Theorem 3.1 were not to occur, then these perturbations could be controlled by requiring that $b_{N} \rightarrow b^{*}$ rapidly enough, $\left\|b_{N}-b^{*}\right\|=o\left(1 / \nu_{N}\right)$, i.e., if the accuracy of measurement and calculation are suitably improved as the approximation is expected to improve.
} 
(2) after the introduction of an approximating parametric form. In the language of statistical practice, such procedures are not robust enough. As is well known, the results are overwhelmingly sensitive to noise in the modes (discarded by the parametrization) associated with eigenvectors of $A A^{*}$ [for this, take $A=f^{\prime}\left(x^{*}\right)$ in the nonlinear case] corresponding to very small eigenvalues. Some desensitization of the computation and the possibility of making use of a priori knowledge of special properties of the solution $x^{*}$ (e.g., extra smoothness beyond membership in $\mathfrak{X}$ ) can be obtained by the use of approximation procedures specifically addressed to the difficulties associated with ill-posed problems (see e.g. Refs. 6 and 7).

\section{References}

1. CANnon, J. R., and Dogru, A. H., Estimation of Permeability and Porosity from Well Test Data, Paper No. SPE-5345, Presented at the 1975 ASME-SPE Meeting, 1975.

2. Chewning, W. C., and Seidman, T. I., A Convergent Scheme for Boundary Control of the Heat Equation, SIAM Journal on Control and Optimization, Vol. 15, pp. 64-72, 1977.

3. GoOdson, R. E., and POLIS, M., Editors, Identification of Parameters in Distributed Systems, American Society of Mechanical Engineers, New York, New York, 1975.

4. NASHED, M. Z., On Moment-Discretization and Least-Squares Solutions of Linear Integral Equations of the First Kind, Journal of Mathematical Analysis and Applications, Vol. 53, pp. 359-366, 1976.

5. RICHTER, G. R., Numerical Solution of Integral Equations of the First Kind with Nonsmooth Kernels, SIAM Journal on Numerical Analysis, Vol. 15, pp. 511-522, 1978.

6. SeIdman, T. I., Convergent Approximation Schemes for Ill-Posed Problems, University of Maryland Baltimore County, Mathematics Department, Research Report No. 78-10, 1978.

7. Tikhonov, A. N., and ARSenin, V. Y., Solutions of Ill-Posed Problems, V. H. Winston and Sons, Washington, DC, 1977. 